\section{Tradición, vanguardia y glocalización: investigación y reflexiones sobre artesanía contemporánea desde la Cátedra de Innovación en Artesanía, Diseño y Arte contemporáneo de la Universidad de Granada}

Ana García López ${ }^{(1)}$

\begin{abstract}
Resumen: La crisis del COVID-19 del año 2020 nos ha posicionado de lleno frente a lo que ya se argumentaba como un cambio de paradigma en el sector de la artesanía que se ve abocado a identificar aquellas oportunidades de desarrollo que ya estaban pendientes de resolver. La investigación desde el estamento universitario es crucial para empujar a la artesanía a la vanguardia, conectando de modo glocal la identidad de las comunidades (pensar global, actuar local), la transdisciplinariedad y la transferencia del conocimiento al tejido social y empresarial, bajo paradigmas de economía circular y sostenibilidad. La Universidad de Granada, con la creación de la Cátedra de Innovación en Artesanía, Diseño y Arte asume la investigación en artesanía aportando un gran nutriente de innovación al sector artesano, en transferencia del conocimiento y en $\mathrm{I}+\mathrm{D}$ desde diversas áreas de conocimiento.
\end{abstract}

Palabras clave: investigación - glocalización - transdisciplinariedad - innovación - transferencia.

[Resúmenes en inglés y portugués en las páginas 56-57]

(1) Ana García López es Doctora en Bellas Artes y profesora en la Facultad de Bellas Artes de la UGR. Realizó el posgrado en "Computer Graphics" en la UCLA (University of California Los Angeles, USA). Es directora de la Cátedra de Innovación en Artesanía, Diseño y Arte Contemporáneo de la Universidad de Granada. Ha sido Vicedecana de Relaciones Internacionales e Investigación de la Facultad de Bellas Artes de la UGR (2008-2018). Su línea principal de investigación se centra en la relación entre arte contemporáneo, diseño, patrimonio y artesanía con el uso de tecnologías, bajo paradigmas de economía circular y sostenibilidad. En la actualidad dirige 3 proyectos de investigación multidisciplinares, multisectoriales e internacionales: dos europeos del programa H2020 (WARMEST y RRREMAKER) y uno nacional de financiación europea FEDER (MakerArt). 


\section{Introducción}

Las crisis traen siempre consigo oportunidades para revisar cuestiones que durante tiempo habíamos creído inamovibles, a pesar de tener certezas o intuiciones previas que ya nos avisaban de necesitar un cambio; el mero hecho de producirse un movimiento en las estructuras y pilares de lo que creíamos estable, facilita la cuestión de repensar, analizar y reorientar, teniendo como oportunidad un momento en que la situación, afectada por un cambio profundo y de consecuencias importantes, se vuelve grave y decisiva hasta el punto de poner en peligro el desarrollo del asunto o proceso del que se trate. Exactamente eso estamos viviendo los habitantes de un mundo globalizado desde los primeros meses de 2020 a causa de la crisis causada por la pandemia que ha desatado el virus COVID-19. Y como todas las crisis, pero aún con más fuerza por la virulencia con la que nos ha penetrado y su globalidad sin parangón, nos ha posicionado abruptamente de frente a lo que ya se venía argumentando como un cambio de paradigma en la manera en la que concebimos nuestra propia existencia, incapaz de adaptarse a las acciones frente a nuestra propia salvación como especie. Queda muy claro que ese cambio radical está afectando a todos los sectores y deberá tener la seguridad humana como objetivo principal, a favor de una profunda transformación socio-ecológica.

De entre todos esos sectores, la oportunidad que se abre ante el sector de la artesanía constituye también un acicate lleno de posibilidades, a pesar de la dureza con la que está golpeando a los habitantes de su frágil ecosistema de pequeñísimas empresas, que ya tenían dificultades previas a esta crisis para subsistir. Sin embargo, las acciones y estrategias hacia las que transitar para salir de ella, generan aportaciones que pueden ser, precisamente, su salvaguarda por ese cambio de paradigma al que ya hemos aludido. El sector se ve abocado a identificar de modo urgente aquellas oportunidades de desarrollo que ya estaban pendientes de resolver y otras nuevas que se derivan de la actual situación, de modo que posibiliten llegar tanto a su público objetivo como a nuevos públicos, a través de la adaptación de los formatos por los que se canaliza, de la propia naturaleza y composición de sus productos, de la adaptación a la economía circular o de los canales de transmisión utilizados. "La artesanía lleva incorporada en su ADN también la resiliencia, la flexibilidad y la agilidad para adaptarse a las circunstancias y situaciones nuevas" (De Dios-Lala, 2020). En este contexto, la investigación y desarrollo que se aporta desde los centros de investigación universitarios a las empresas se va a configurar como elemento clave de este impulso, ya que la propia naturaleza de la artesanía proporciona un rico campo transdisciplinar de estudio apenas explorado por la alta investigación aplicada. Por tanto, se vislumbra como un escenario en el que las muchas áreas que conforman la estructura de investigación de un territorio ligada a sus estamentos universitarios pueden y deben ofrecer al sector nuevo conocimiento para aplicar a su desarrollo. Ese desarrollo puede impulsar a la artesanía a la vanguardia y ayudarla a establecer transiciones que van a ser cruciales en su desarrollo y avance, tales como la transición a la economía circular, la utilización de tecnologías y la adaptación a un mercado que ha cambiado desde la aparición del virus, por nombrar algunas claves.

No obstante, es necesario no perder de vista la naturaleza del sistema de valores sobre los que la artesanía se ha construido a lo largo del tiempo, pues es importante que la 
aportación que se produzca desde la investigación se desarrolle bajo la comprensión del territorio sobre el que se han construido las mimbres de dicho sector, pues en su mayor parte, se basa en una tradición heredada que a su vez mantiene especiales vínculos con el territorio en el que se desarrolla. Se trata al fin de un compendio de saberes, técnicas, procesos y procedimientos que dan lugar a objetos bien identificables, y este patrimonio, si no se trata con delicadeza, es fácilmente pervertible, por lo que las aportaciones que se produzcan para alentar su desarrollo y abordar los desafíos que enfrenta, podrían acabar siendo su definitiva puntilla en lugar de ser sus impulsoras.

Por tanto, las estrategias que desde la investigación se aporten a través de sus áreas de conocimiento deben aplicarse conectando la identidad de las comunidades y los saberes ancestrales de los que se parte ese patrimonio intangible que la UNESCO define bien como patrimonio cultural inmaterial y en la que incluye los saberes y técnicas vinculados a la artesanía tradicional. Pese a su fragilidad, el patrimonio cultural inmaterial es un importante factor del mantenimiento de la diversidad cultural frente a la creciente globalización. La comprensión del patrimonio cultural inmaterial de diferentes comunidades contribuye al diálogo entre culturas y promueve el respeto hacia otros modos de vida. La importancia del patrimonio cultural inmaterial no estriba en la manifestación cultural en sí, sino en el acervo de conocimientos y técnicas que se transmiten de generación en generación, según la UNESCO.

\section{De la glocalización al diseño distribuido y el I+D aplicado: el poder de las comunidades}

Algunas de esas estrategias parten de la filosofía de la glocalización y aluden a la transdisciplinariedad, la co-creación, la transferencia del conocimiento al tejido social y empresarial o la utilización de tecnologías bajo paradigmas de economía circular y sostenibilidad.

Pensar globalmente y actuar localmente es la base de la filosofía de la glocalización, acrónimo formado por las palabras globalización y localización, acuñado por primera vez por el sociólogo Ronald Robertson (2003) que la define como un concepto "que se refiere tanto a la compresión del mundo y a la intensificación del mundo como un todo" (Robertson, 2000). La antropóloga del arte Lourdes Méndez, distingue dos dinámicas inseparables en el mundo actual: la globalización y la glocalización, siendo la primera el proceso acelerado de interdependencia en el mundo actual mientras que la segunda sería la interpretación entre lo global y local, teniendo en cuenta que cada comunidad humana puede formar parte de la globalización manteniendo una identidad cultural específica (García-Mazuecos, 2017). La forma de trabajar de los artesanos está muy conectada con paradigmas que tienen que ver con la co-creación y el diseño distribuido. Frente a una visión romántica de los artesanos, como especímenes de otra época trabajando en sus talleres de forma aislada, hay un grupo de consumidores que miran a la artesanía de un modo más contemporáneo, en el que la creación a mano no está reñida con la utilización de tecnologías y en la que nos encontramos productos que ofrecen no sólo un modo de creación emocional, sino también una actitud responsable en cuanto a los parámetros de sostenibilidad y ecodiseño. 
En realidad, esta forma de mercado define la estrategia de grandes empresas que adaptan sus productos a un contexto local. Sin embargo, nuestra propuesta es adaptar esta corriente para centrarse precisamente en cómo pequeñas empresas artesanas son capaces de llevar sus relatos desde lo local hasta el mercado global y hacer que trascienda la barrera del mercado local abordando a nuevos públicos sin que por ello el producto sufra cambios en su conceptualización, diseño y fabricación. "La artesanía está descubriendo nuevas identidades, está dialogando entre lo local y lo global, entre la tradición y la innovación, entre lo masculino y lo femenino, está explorando valores más humanos, un equilibrio entre ecología, sostenibilidad y desarrollo" (Santos Capa, 2020). El concepto se vincula a lo que se ha dado en llamar diseño distribuido donde viajan globalmente los datos (diseño) mientras que los átomos (material) permanecen en el entorno local utilizando los beneficios de una sociedad globalmente conectada para mover datos en lugar de productos. Este paradigma presenta una nueva alternativa sostenible al modelo industrial de "tomar, hacer, desperdiciar" y los diseñadores pueden crear productos personalizados y localizados en colaboración con otros a distancia ${ }^{1}$.

El diseño distribuido es un modelo que apuesta por el diseño global compartido que puede cambiar el actual sistema de producción reduciendo el impacto ecológico de los productos, democratizando el acceso a un diseño de calidad y ampliando el mercado para artesanos, diseñadores y consumidores. Volvemos a hablar de oportunidades pues las nuevas tecnologías de fabricación digital y diseño de materiales ofrecen estrategias muy valiosas para repensar el presente a través de la artesanía.

En la actualidad compramos productos que viajan miles de kilómetros para llegar a nosotros, igualmente la materia que los compone recorre grandes distancias antes de llegar a la fábrica. Es en este contexto en el que la artesanía local se convierte en un motor de cambio y se beneficia del trabajo en red, al compartir herramientas y ser más accesible a sus consumidores. Se trata de abrir los talleres al espacio social que se ha visto implementado por las tecnologías de manufactura avanzada y los nuevos formatos de comunicación. Esta manera de actuar tiene mucho que ver con las personas, con las comunidades y los lugares, pues las conexiones y las habilidades ayudan a desarrollar la creatividad que es el asunto central y fundamental del impulso.

En este contexto se ha desarrollado la plataforma Maker $\mathrm{Art}^{2}$ dentro del proyecto de Investigación y Desarrollo Maker Art: una propuesta para la transformación digital de la industria cultural relacionada con la artesanía, a través del diseño, los procesos colaborativos y la cultura Maker que fue financiado por fondos FEDER dentro del reto "Economía y Sociedad Digital / Sociedades inclusivas, innovadoras y reflexivas" a través de la convocatoria de la Junta de Andalucía en la convocatoria de 2018 y se está desarrollando hasta 2022. El planteamiento es el de la creación de una plataforma de intercambio que es punto de encuentro de comunidades de productores de contenidos, donde se incluyen artesanos tradicionales, como los que empiezan a integrar tecnologías en su trabajo o los que realizan labores de producción y diseño con medios digitales. La plataforma plantea oportunidades de colaboración a partir de la puesta en común de diseños y metodologías de producción que incorporen tecnologías maker, prácticas de comunicación y gestión de conocimiento, y modelos de negocio que es punto de encuentro de comunidades de productores de contenidos, donde se incluyen tanto artesanos tradicionales, como los que 
empiezan a integrar tecnologías en su trabajo o los que realizan labores de producción y diseño con medios digitales. La plataforma plantea oportunidades de colaboración a partir de la puesta en común de diseños y metodologías de producción que incorporen tecnologías maker, prácticas de comunicación y gestión de conocimiento y modelos de negocio que aprovechen las posibilidades del entorno digital.

Dentro de la puesta en común de diseños y metodologías de producción se considera la implementación de un canal directo de intercambio de archivos de diseños entre agentes procedentes de cualquier parte del mundo. Entre otras múltiples posibilidades, por ejemplo, el artesano podrá crear el objeto artesanal con técnicas propias de su oficio a partir del prototipo producido mediante impresión aditiva u otros métodos de reproducción digital. Así, a través de la colaboración y mediante el intercambio de prototipos en red, se pretende optimizar la producción y actualizar los diseños, produciendo un impacto en la modernización del sector, con repercusión en la industria cultural y turística, ofreciendo oportunidades de innovación social y emprendimiento para las nuevas generaciones.

La idea principal de la plataforma Maker Art es conectar con otras comunidades a través de recursos existentes como plataformas de diseño distribuido, Fab labs y espacios digitales de comunicación global entre comunidades locales y continuar contribuyendo a ese ecosistema de plataformas donde se comparte conocimiento y contenido.

Una de las externalidades positivas que ha emanado de este proyecto ha sido la idea de perfeccionar esta plataforma de encuentro e intercambio, implementando un sistema que aportara otros beneficios más allá de los descritos. Uno de los condicionantes que nos impusimos como grupo de investigación fue el de perfeccionar y aumentar las capacidades de la plataforma de forma que pudiera ayudar a la artesanía a transitar desde un modelo de economía lineal hacia un modelo de economía circular. Los oficios artesanales y su técnicas y metodologías cobran vigencia en un mundo donde la economía circular marca tendencia como nuevo modelo: reutilizar, reciclar y reducir es la consigna (García, 2021). Por otro lado, nos planteamos la necesidad de que la propia plataforma no fuera un actor pasivo y tuviera la capacidad de ofrecer opciones al artesano, maker o diseñador de modo que supusiera un banco de posibilidades con los que trabajar para ofrecer productos competitivos en un mercado en pleno proceso de transición. Esta doble estrategia, la de la sostenibilidad más la del diseño se formuló atendiendo al diseño generativo y la utilización de la inteligencia artificial. El resultado fue la formulación del proyecto de investigación llamado RRREMAKER en el que el objetivo ha sido establecer un nuevo modelo de gestión híbrido basado en las comunidades de conocimiento, el ecodiseño, integrando paradigmas de economía circular, economía creativa y economía colaborativa (sharing economy $)^{3}$. La innovación de este proyecto radica en el diseño y desarrollo de esta plataforma basada en Inteligencia Artificial pues se convertirá en un actor esencial en sí mismo en lo que respecta al proceso creativo, ya que aportará posibilidades casi infinitas al creador a través de la integración de algoritmos de vanguardia de diseño generativo, algoritmos genéticos y de base supercuádrica, clasificadores de aprendizaje automático (machine learning), algoritmos para big data, computación en nube y datos experimentales; todos ellos ofrecerán predicciones acerca de estructuras, formas, materiales, colores, ornamentación, etc., basados en parámetros de diseño artesanales tanto tradicionales como innovadores y provenientes de materiales de reciclaje. El proceso de esta plataforma no es absolutamente 
automático pues estará controlado por el artesano, el diseñador o el productor (maker) que podrá aplicar restricciones y limitaciones al diseño.

El proyecto RRREMAKER (Reutilizar, Reducir, REciclar: plataforma basada en Inteligencia Artificial para la fabricación automatizada y escalable en la cultura MAKER bajo parámetros de Economía Circular) se ganó en la convocatoria de proyectos de I+D H2020 de la EU con un presupuesto de 1,5 M.€ y ha supuesto la creación de un consorcio de 7 empresas y 3 universidades de 6 países de Europa y 1 de América Latina con investigadores expertos en ingeniería, economía, bellas artes y patrimonio, comunicación, ciencia de datos, marketing, sostenibilidad y nuevos materiales que, desde enero de 2021 hasta diciembre de 2024, van a trabajar para perseguir este ambicioso reto en un programa de investigación conjunta y transferencia del conocimiento a través de estancias de investigación financiadas. Los participantes en el proyecto, PYMES e instituciones europeas y latinoamericanas, intercambiarán competencias y conocimientos que les permitirán progresar hacia avances clave en una solución real para integrar la economía circular en la artesanía, hasta la introducción de una nueva forma de "fabricación inteligente" extendida a una cadena de producción más amplia y multicultural y compuesta por recolectores de residuos, diseñadores y ecodiseñadores, laboratorios de prototipos rápidos y artesanos. En este marco se introducirá el concepto de nutriente tecnológico (Molina-Moreno; Leyva-Diaz; Sanchez-Molina, 2016, p. 777) que convierte un recurso ya utilizado en alimento para otro proceso para definir la unidad de información de diseño único.

El ecodiseño como estrategia y herramienta para transformar a través de la innovación los procesos de creación y de consumo en sostenibles, se hace indispensable en el camino del artesano contemporáneo pues prioriza la reducción total del impacto ambiental. Sólo así será el sector artesanal capaz de seguir pautas eficientes en el uso de recursos en línea con los principios de la economía circular. Sólo así pervivirá la artesanía contemporánea, estableciendo un estrecho vínculo entre tradición y contemporaneidad, entre local y global, con la estética ecológica como marco, promoviendo la práctica y educación sobre el respeto y la recuperación del medioambiente.

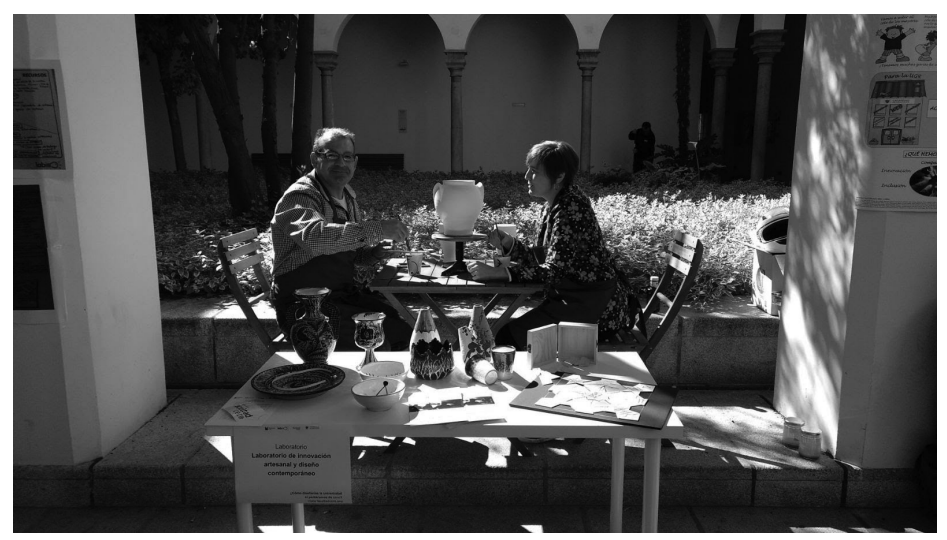

Imagen 1.

Presentación del Laboratorio de Innovación Artesanal y Diseño contemporáneo en el Proceso Facultad Cero. 


\section{La creación de la Cátedra de Innovación en Artesanía, Diseño y Arte Contemporáneo de la Universidad de Granada}

Desde 2007 establecimos una línea de investigación que conecta el patrimonio y las nuevas tecnologías para abordar estrategias de impulso, comunicación, predicción y salvaguarda de este. Dichas líneas se han establecido desde el inicio en sinergia con instituciones y empresas dentro y fuera del país, aportando a la investigación una dimensión intersectorial e internacional que enriquece la calidad de los resultados y la transferencia del conocimiento al tejido empresarial. En la actualidad dirigimos proyectos de investigación, desarrollo e innovación que indagan en procedimientos, técnicas y materiales tradicionales utilizados por artesanos con materiales autóctonos o reciclados, con el fin de recuperarlos para el mercado actual, rediseñando formas, color, materiales y posibles usos, estableciendo puentes entre arte, artesanía, ecología y economía, tecnología y tradición, que ayuden al sector artesanal a ofrecer productos competitivos en un mercado en pleno proceso de transición.

Otros proyectos menores pero de importancia han reflejado la línea de investigación establecida, como la creación del UGR ArtLab Laboratorio de Innovación Artesanal y Diseño contemporáneo ${ }^{4}$ (en el marco del proyecto Facultad Cero del Media Lab de la Universidad de Granada) o los encuentros A+D+A (Artesanía+Diseño+Arte) en 2018 y 2020, como encuentro entre diseñadores, artistas y artesanos que trabajaron juntos en los talleres para producir prototipos ligados al imaginario de la ciudad de Granada y su patrimonio (Edición de 2028) y como espacio de reflexión en pleno año COVID para repensar la artesanía y proponer estrategias desde diferentes estructuras y disciplinas (Edición de 2020)

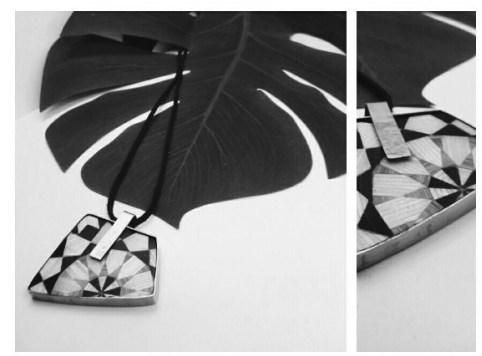

2

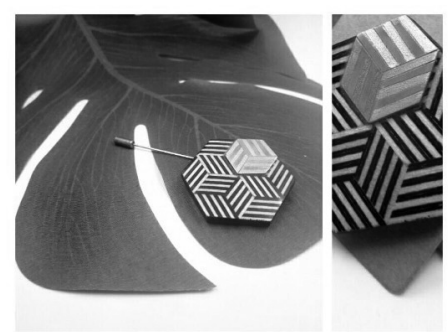

3

Imagen 2 y 3. Oscar Beas (Artesanías Beas) y Rocío Campaña (Diseñadora). Presentación del prototipo de joya a partir de taracea granadina durante los encuentros ADA 2018. 
A partir de las sinergias establecidas con el Centro de Referencia Nacional de Artesanía (sito en la ciudad de Granada en uno de los barrios más vinculados con la artesanía como es el Albayzín) y el marco de proyectos de investigación presentados, se creó el tejido investigador propicio y suficiente como para que en 2020 la Universidad de Granada auspiciara la creación de la que es la primera Cátedra de Innovación en Artesanía, Diseño y Arte contemporáneo de una universidad española, que inició su andadura en enero de 2021.

Desde fuera de las estructuras universitarias, cabría preguntarse ¿por qué una cátedra? ¿Qué aporta a la sociedad en general y a los sectores implicados en particular? La respuesta la trataremos de dar en estas líneas. La estructura de cátedras de la Universidad de Granada entronca con su política de mantener relaciones con su entorno local, nacional e internacional, manteniendo actividades de colaboración con instituciones y empresas con el objetivo de beneficiarse mutuamente de los resultados de investigación, el desarrollo y la innovación que derivan de la actividad universitaria para contribuir al progreso social y desarrollo económico de la sociedad (art. 194 de los estatutos de la UGR). En este contexto la universidad amplía los instrumentos de colaboración más allá de los convenios o los contratos de investigación, al regular una forma distinta de interacción con la sociedad en forma de cátedras, que son estructuras que se conciben como alianzas estratégicas estables con empresas e instituciones públicas o privadas para el desarrollo de actividades de formación, de generación, divulgación o transferencia del conocimiento en una determinada área, contando con la financiación de dichas empresas o instituciones, que se benefician de su acercamiento a la investigación y al conocimiento generado en la universidad ${ }^{5}$.

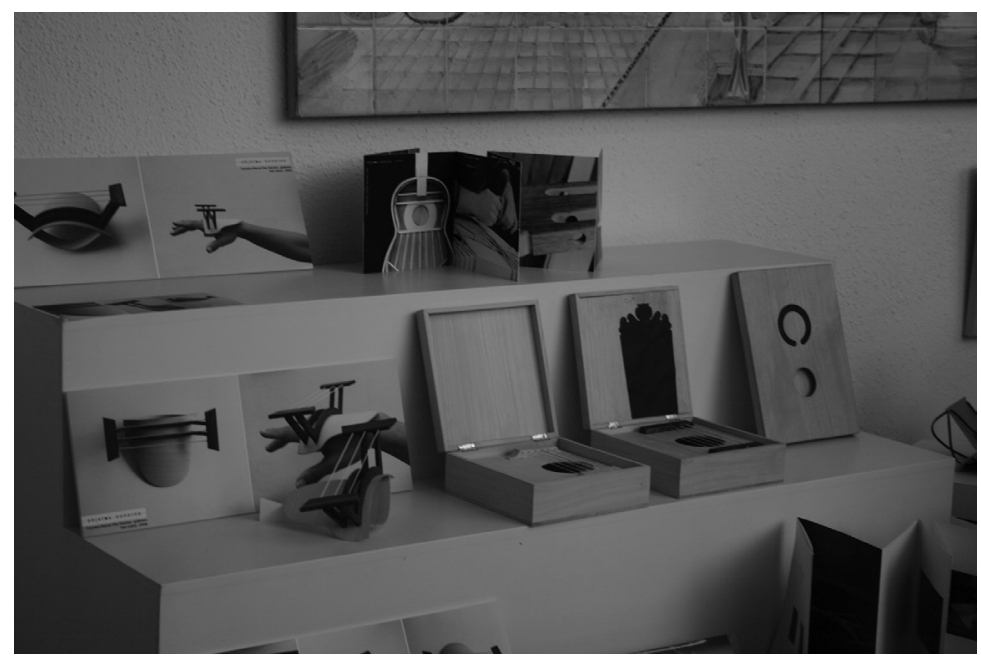

Imagen 4. Oscar Beas (Artesanías Beas) y Rocío Campaña (Diseñadora). Presentación del prototipo de joya a partir de taracea granadina durante los encuentros ADA 2018. 
La Universidad de Granada, con la creación de la Cátedra de Innovación en Artesanía, Diseño y Arte asume la investigación en artesanía aportando un gran nutriente de innovación al sector artesano, en transferencia del conocimiento y en I+D desde las Artes y el Diseño, desde la Economía y el Mercado, la Ingeniería, el Derecho o la Comunicación, entre otros ámbitos del conocimiento. Esta Cátedra es producto del convenio firmado entre la UGR con el Centro de Referencia Nacional de Artesanía / Centro Albayzín con el que ya había un amplio y fructífero historial de cooperación en materia de formación, producción, investigación y difusión alrededor de la artesanía. El CRN Albayzín depende del Ministerio de Educación de España y del Servicio Andaluz de Empleo del Gobierno andaluz (Junta de Andalucía), por lo que el convenio que dio lugar a la cátedra se firmó con este. Es destacable la doble naturaleza que subyace al CRN y las grandes ventajas que ofrece pues su dedicación a la formación (Ministerio) y a las políticas de empleo (Junta de Andalucía) quedan indefectiblemente reforzadas a través de las estrategias de investigación y formación que se ofrecen desde el estamento universitario a través de la Cátedra. Entre los fines del Centro Albayzín como CRN de Artesanía ${ }^{6}$ se encuentran el análisis de nuevas tendencias formativas, su experimentación y puesta en práctica, y la promoción de redes tanto con organizaciones empresariales y sindicales como con universidades y centros tecnológicos con la finalidad de ser puntero en el sector productivo relacionado con las familias y áreas profesionales de referencia, dando prioridad a atender las necesidades de los sectores emergentes e innovadores y conseguir así una formación profesional más competitiva, que responda a los cambios productivos que demanda el mercado de trabajo. Además, en relación directa con el objeto de convenio para el establecimiento de la Cátedra, el CRN de Artesanía tienen entre sus funciones: observar y analizar, a nivel estatal, la evolución de los sectores productivos, para adecuar la oferta de formación a las necesidades del mercado de trabajo; elaborar contenidos, metodologías y materiales didácticos, fomentar la investigación, innovación y desarrollo de la formación; observar y analizar la evolución de las bases científicas y tecnológicas relacionadas con los procesos de formación o con el sector de referencia; así como participar en programas e iniciativas internacionales en su ámbito de actuación; todas ellas funciones que se corresponden estrechamente con los fines de la Universidad de Granada directamente relacionados con la artesanía.

Por tanto, la cátedra brinda una oportunidad amplia a través de la colaboración basada en la creación de programas de actualización y formación en los grados, tercer ciclo y doctorados. Igualmente, las prácticas externas de estudiantes de los grados universitarios en el propio CRN y en pequeñas y medianas empresas artesanas es crucial pues se genera una relación directa entre los trabajos y proyectos de fin de Grado, Trabajos Fin de Máster donde aplicar conocimientos que provienen de muchas titulaciones, pero sobre todo desde las Artes, Diseño, Mercado, Ingenierías, Economía, Derecho, etc. Además, las acciones de observación, investigación y transferencia de resultados de investigación a través de proyectos de investigación, tesis doctorales, grupos y trabajos de investigación vinculados a la cátedra estableces parámetros y ofrecen nuevo conocimiento en la observación de la evolución de los sectores productivos, en las bases científicas y tecnológicas relacionadas con los procesos de formación o con el sector. De este modo se procura la mejora de la formación y la empleabilidad en sectores vulnerables de la población, asumiendo un trabajo 
de cooperación con empresas y entidades públicas y privadas para el desarrollo de estrategias que faciliten la adaptación de personas con discapacidad en el sector de la artesanía.

\section{Conclusiones}

El desafío que supone al sector artesanal sobrevivir a la crisis de COVID en un mundo post pandemia plantea retos que bien pueden ser tomados como oportunidades para revisar las claves sobre las que se sustenta la supervivencia del sector. Es necesario tomar esta crisis como una oportunidad bajo los nuevos parámetros que rigen la estructura y organización del trabajo que va a tener éxito en este nuevo escenario.

Es clave asumir que la conexión entre las comunidades a escala global y el fomento del trabajo en red ayuda a reforzar la identidad local y el tejido empresarial ligado al territorio a base de compartir herramientas, conocimiento y soluciones que son el sustento de la co-creación y el diseño distribuido. Entre las estrategias que están llamadas a impulsar la artesanía contemporánea, no se puede pasar por alto la aplicación de tecnologías ligadas a ramas de la ingeniería como son la robótica, la inteligencia artificial, el aprendizaje automático o machine learning, la ingeniería de materiales o el diseño generativo, que pueden aportar una gran riqueza de posibilidades a los artesanos. Junto con la implementación de estrategias basadas en las tres Rs (Reciclar, Reutilizar, Reducir) el artesano será capaz de navegar y orientarse en la ideación de nuevas formas, con nuevos materiales y procesos provenientes de la reutilización de materiales. Estas estrategias, lejos de usurpar su lugar como homo faber, ayudarán a los artesanos a avanzar hacia un entorno más favorable para construir un modo de vida basado en la riqueza de la producción artesanal de vanguardia, donde la contemporaneidad no esté reñida con la tradición, ni lo local con lo global (García, 2020).

La investigación aplicada a través de líneas conectadas con las distintas áreas de conocimiento desde las instituciones universitarias públicas y privadas se muestra como pieza clave para aportar un rico nutriente de conocimiento y aplicación de recursos y estrategias para llevar a la artesanía contemporánea desde la tradición a la vanguardia en procesos, producto y comercialización.

\section{Notas}

1. Distributed Design. FabLab Barcelona. https://fablabbcn.org/projects/distributed-design

2. https://makerart.si2.ninja

3. La economía compartida, que ha crecido exponencialmente en los últimos años con plataformas como AirBnb o Uber, se define como el paradigma bajo el cual los modelos de negocio verticales, jerárquicos y centralizados, se transforman en modelos horizontales, colaborativos y comunitarios. Para ello, la economía colaborativa procura la creación y el desarrollo de infraestructuras digitales y físicas que facilitan el intercambio entre indi- 
viduos ("Peer-to-Peer"). Es imprescindible subrayar que al sharing economy le subyacen una serie de principios y valores cuyo eje central está compuesto por la cooperación, el desarrollo económico local y sustentable, y la responsabilidad social. Ver en Rodriguez Lopez, A. (2019) Colmena66 Team. https://www.colmena66.com/en/blog/blog/2019/04/25/ qué-es-la-econom\%C3\%ADa-colaborativa-o-sharing-economy (Acceso 5 de Octubre 2020). Ver también en Janelle Orsi, Practicing Law in the Sharing Economy: Helping People Build Cooperatives, Social Enterprise, And Local Sustainable Economies (2012); Rachel Botsman \& Roo Rogers, What's Mine Is Yours: The Rise Of Collaborative Consumption (2010); Arun Sundararajan, The Sharing Economy: The End of Employment and the Rise of Crowd-Based Capitalism (2017);

4. Recuperado el 22/02/2021 de: https://facultadcero.org/2018/05/15/propuesta-laborato rio-art-lab/

5. Normativa para la creación de cátedras y aulas institucionales y de empresa de la Universidad de Granada. https://www.ugr.es/sites/default/files/2018-01/NCG1271.pdf

6. Real Decreto 502/2019, BOE no221 de 14 /09/2019. https://www.boe.es/boe/dias/2019/ 09/14/pdfs/BOE-A-2019-13091.pdf

\section{Bibliografía y referencias}

Anderson, C. (2013). Makers. La nueva revolución industrial. Barcelona: Urano.

Braungart, M.; McDonough, W. \& Bollinger, A. (2007). "Cradle-to-cradle design: creating healthy emission- a strategy for eco-effective product and system design” en Journal of Cleaner production. Pp. 1337-1348.

Briceño, A. (2018). “Escenarios distribuidos, Albores de un nuevo modelo”. Recuperado el 20/03/2021 en http://www.fundaciondid.cl/el-nuevo-modelo-albores-de-una-nueva-re volucion-industrial-por-andres-briceno/

De Dios, L. Encuentros ADA 2020 "Impulsar la artesanía”. Recuperado el 21/02/2021 de https://www.youtube.com/watch?v=XZ64jZ_lNK0\&t=467s

García-Lopez, A. (2021). “Nuevos paradigmas para la artesanía contemporánea en el contexto de la investigación en economía circular” en Meseguer Sánchez, J. V. et Alt. (2021) Economía Circular: fundamentos y aplicaciones. Pamplona: Thomson Reuters Aranzadi. . (2020). "La investigación como estrategia para impulsar la artesanía" en Garcia, A. y Suarez, A. (2020) Repensar la Artesanía: estrategias para impulsar la artesanía contemporánea. Granada: Comares.

Ghelfi, D. (2005). Understanding the engine of creativity in a creative economy: an interview with John Howkins. World intellectual property organization, Geneva. Recuperado el 12/04/2021 de https://www.culturenet.cz/coKmv4d994Swax/uploads/2018/08/Unders tanding-the-Engine-of-Creativity-in-a-Creative-Economy.pdf

Howkinks, J. (2013). The Creative Economy: how people make money from ideas. Londres: Penguin. ISBN 0141977034.

Meseguer Sánchez, J. V. et Alt. (2021). Economía Circular: fundamentos y aplicaciones. Pamplona: Thomson Reuters Aranzadi. 
Molina-Moreno, V.; Leyva-Diaz, J. C. \& Sánchez-Molina, J. (2016). “Pellet as a technological nutrient within the circular economy model: comparative analysis of combustion efficiency and $\mathrm{CO}$ and $\mathrm{NO}_{\mathrm{x}}$ emissions for pellets from olive and almond trees". Energies 9, 777.

McCullough, M. (1996). Abstracting Craft: the Practiced Digital Hand. Cambridge: MIT Press, MA.

Robertson, R. (2003). "Glocalización: tiempo-espacio y homogeneidad-heterogeneidad" en Cansancio del Leviatán: problemas políticos de la mundialización. Madrid: Trotta. 8481646253.

Robertson, R. (2000). “Glocalización: tiempo-espacio y homogeneidad-heterogeneidad”. Zona Abierta 92-93.

Santos Capa, J. C. Encuentros ADA 2020 “Impulsar la artesanía”. Recuperado el 21/02/2021 de https://medialab.ugr.es/noticias/ii-jornadas-ada-segunda-sesion/

VVAA_Guía de recomendaciones y buenas prácticas. Producción y consumo de productos artesanos sostenibles, publicado por el Instituto para el fomento del desarrollo y la formación. Recuperado el 24/10/2020 de: https://drive.google.com/file/d/1OqfLwBEYnm M4ArQU0egnMMWgAXOOvdwD/view

VVAA_Report on the situation of craftsmanship in Europe. CR@FTSMAN PROJECT ES/09/ LLP-LdV/TOI/149072. Recuperado el 24/10/2020 de: http://projects.ifes.es/pdfs/craft/ craft1.pdf

VVAA_II Plan integral para el fomento de la artesanía en Andalucía (2014-2017). Recuperado el 12/10/2020 de: http://www.juntadeandalucia.es/turismoycomercio/publicaciones/ 143474860.pdf

\begin{abstract}
The COVID-19 crisis of 2020 has positioned us squarely in front of what was already argued as a paradigm shift in the craft sector that is forced to identify those development opportunities that were already pending to be resolved. Research from the university is key to push the craft to the forefront, connecting in a glocal way the identity of communities (think global, act local), transdisciplinarity and knowledge transfer to the social and business fabric, under paradigms of circular economy and sustainability. The University of Granada, with the creation of the Chair of Innovation in Crafts, Design and Art, assumes the research in crafts, providing a great source of innovation to the crafts sector, in knowledge transfer and R\&D from different areas of knowledge.
\end{abstract}

Keywords: research - glocalization - transdisciplinarity - innovation - knowledge transfer.

Resumo: A crise do COVID-19 de 2020 nos colocou diretamente frente ao que já foi argumentado como uma mudança de paradigma no setor artesanal que visa identificar as oportunidades de desenvolvimento que já estavam pendentes de serem resolvidas. A investigação a partir do nível universitário é fundamental para colocar o artesanato em primeiro plano, ligando de forma glocal a identidade das comunidades (pensar global, agir local), a transdisciplinaridade e a transferência de conhecimento, para o tecido social 
e empresarial, sob os paradigmas da economia circular e da sustentabilidade. A Universidade de Granada, com a criação da Cátedra de Inovação em Artesanato, Design e Arte, assume a investigação em artesanato, contribuindo com uma grande fonte de inovação para o sector artesanal, na transferência de conhecimentos e na I+D de diferentes áreas do conhecimento.

Palavras chave: pesquisa - glocalização - transdisciplinaridade - inovação - transferência.

[Las traducciones de los abstracts fueron supervisadas por el autor de cada artículo] 